\section{DNA databases shut after identities compromised}

Several DNA databases run by the US National Institutes of Health (NIH) in Bethesda, Maryland, the Wellcome Trust in London and the Broad Institute in Cambridge, Massachusetts, were closed to public access last week after researchers showed it is possible to extract the supposedly confidential identities of the patients involved. The databases list the frequencies of small DNA variations called single nucleotide polymorphisms (SNPs) from patient groups.

In the August issue of PLoS Genetics, Nils Homer and his colleagues describe a method to mine individual SNP profiles from complex mixtures, even if the person's DNA is only $0.1 \%$ of the total. The method could be useful for ensuring patients are not listed twice when scientists combine data sets, as well as in forensic science.

The NIH has not identified any patient privacy violations, and points out that to identify a particular patient, one would need his or her genetic profile. Researchers will now have to apply for data access at the individual level, as they do for study data.

\section{Bubble-fusion researcher loses misconduct appeal}

Nuclear engineer Rusi Taleyarkhan has been stripped of his named professorship at Purdue University in West Lafayette, Indiana, following the results of a misconduct inquiry into his bubble-fusion research.

Having lost an appeal against the university's misconduct ruling, Taleyarkhan is banned from having graduate students for three years, and loses the title 'Arden L. Bement Jr Professor of Nuclear
Engineering, along with annual resources of $\$ 25,000$ that come with it. He will, however, remain on the faculty and have his situation reviewed in three years.

According to an investigation report released on 18 July, Taleyarkhan's misconduct involved two falsifications of the research record. In a recent e-mail to Nature, Taleyarkhan denies both charges, calling the findings "grossly inappropriate" and adding, "the two allegations for which misconduct was concluded have nothing to do with the science of bubble nuclear fusion". He also questions the sanctions, given that "a duly constituted committee in 2006 looking at these same two issues" exonerated him. For a longer version of this story, see http://tinyurl. com/5mqyhn

\section{'No pollution effects' from Chinese chemical explosion}

An explosion that killed 20 people last week at a chemical plant in Yizhou, in the southern Chinese province of Guangxi, poses no further threat, according to preliminary surveys by the Chinese health ministry.

The cause of the 26 August explosion is under investigation. Liu Xiongmin, an engineer specializing in disaster management at Guangxi University in Nanning, says the explosion may have been caused by a chemical leak coupled with very hot temperatures that day, which reached $36^{\circ} \mathrm{C}$. The plant produced polyvinyl acetate, carbide and vinyl acetate.

The explosion destroyed the five-storey plant as well as nearby houses. Liu says that of the bulk chemicals used at the plant, ethanol was mostly burned in the explosion and methyl alcohol does not seem to have escaped in large amounts. Reportedly, none of the 60 people injured in the blast had toxic reactions.

\title{
Mars rover climbs out of crater to focus on plains
}

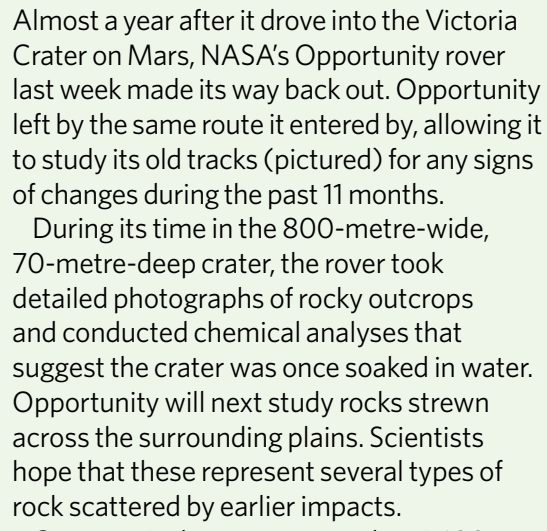

martian days exploring and is showing its age. Its robotic arm is kept permanently extended owing to fears about the health of its 'shoulder' motor, and engineers recently noted an electric-current spike similar to that seen in Opportunity's twin rover Spirit shortly before it lost the use of one of its wheels.

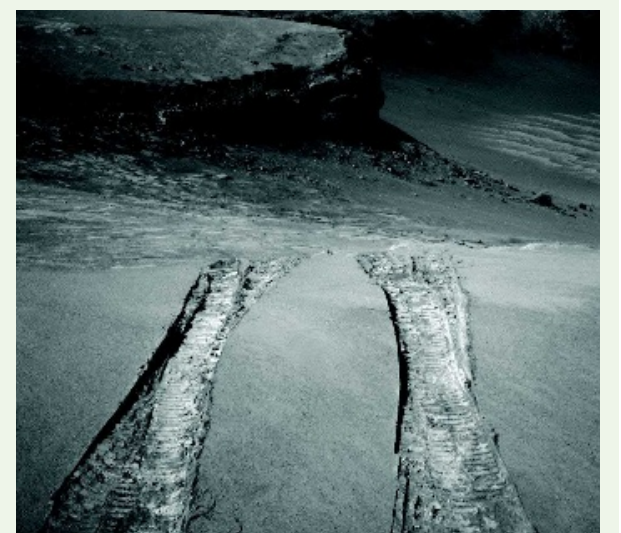

The Journal of Visualized Experiments (JoVE) has announced that its online video protocols will be indexed in the popular US National Library of Medicine repositories MEDLINE and PubMed.

Founder and chief executive Moshe Pritsker views the MEDLINE-PubMed listing as a sign that the scientific community has accepted video-based publications. "It was a very important decision for us, and for scientific publishing," he says.

Since JoVE was founded in 2006 with support from an angel investor, the journal has published more than 200 videos, most produced by professional videographers. It aims to improve the reproducibility of scientific results by using videos to clarify subtle experimental details. The journal was itself an experiment in video publishing and remains the only videobased scientific journal. 